Trauma Berufskrankh 2014 • 16[Suppl 2]:167-168 DOI 10.1007/s10039-013-1997-7

Online publiziert: 14. September 2013

c) Springer-Verlag Berlin Heidelberg 2013
J. Kolbenschlag $\cdot$ M. Lehnhardt

Klinik für Plastische Chirurgie und Schwerbrandverletzte, Handchirurgiezentrum, Referenzzentrum

für Gliedmaßentumoren, BG-Universitätsklinikum Bergmannsheil, Ruhr-Universität Bochum

\section{Funktionelle Rekonstruktion peripherer Nerven}

\section{Bedeutung der End-zu-Seit-Neuroraphie}

Die Technik der End-zu-Seit-Neuroraphie stellt eine effektive Alternative zu den in der peripheren Nervenrekonstruktion etablierten Verfahren wie der End-zuEnd-Koaption mit Spendernerven oder der Nerveninterposition bei Substanzverlust durch Kabelinterponate dar. Technisch wird bei fehlendem proximalem Nervenstumpf der distale Nervenanteil an einen gesunden Spendernerven koaptiert, um auf diese Weise sowohl eine sensorische als auch eine motorische Reinervation zu erhalten.

\section{Historie}

Die Möglichkeit der End-zu-Seit-Neuroraphie wurde erstmals 1873 durch Letievant [4] beschrieben und 1903 durch Ballance et al. [1] erstmalig am Patienten eingesetzt. Ballance et al. [1] nahmen bei Vorliegen einer Fazialisparese die Koaption des distalen Fazialisanteils auf den N. accessorius vor. Da bei absolut erfolgreichem Ergebnis jedoch eine gemeinsame Bewegung von Schulter, Zunge und Gesicht resultierte, wurde diese Technik allgemein abgelehnt. Zusätzlich ergab sich der Nachteil, dass im Bereich des Spendernervs aufgrund der vorgenommenen Durchtrennung einzelner Axone ein funktionelles Defizit hingenommen werden musste.

Erst 1992 wurde die Technik durch Viterbo et al. [6] experimentell und klinisch wiederaufgenommen. Experimentell wurde bei Ratten der N. peronaeus durchtrennt und dessen distaler Anteil an den N. tibialis koaptiert. Klinisch wurden durch unterschiedliche Arbeitsgruppen v. a. bei der Reanimation des gelähmten Gesichts durch Koaption des N. facialis auf den N. hypoglossus Erfolge verzeichnet. Aber auch im Rahmen von Dupuytren-Operationen, bei Affektionen des R. superficialis oder Handverletzungen, bei denen der distale Stumpf des N. ulnaris auf den n. medianus genäht wurde, zeigten sich Erfolge, die den anderen Techniken bis hin zur Primärnaht des Nervs vergleichbar waren [3].

Zuvor jedoch wurde die Technik fundamental weiterentwickelt: Da Verluste im Bereich des Spendernervs nicht hingenommen werden konnten, erfolgte zunächst die End-zu-Seit-Koaption an den Donornerven ohne eine Präparation des Spenders, das Peri- und Epineurium wurden dabei nicht verletzt. Diese Hüllschichten jedoch erwiesen sich als Hürde, die von den sprießenden Axonen nicht überwunden werden konnte. Erst die Eröffnung des Epineuriums erbrachte den gewünschten Erfolg. Reinervationsraten von bis zu 90\% konnten erreicht werden, ohne dass der Spendernerv tangiert wurde. Das Sprießen und Vorwachsen von Axonen des Spendernervs in den distalen Stumpf konnten dabei nachgewiesen werden. Aktuelle Literaturrecherchen $[5,7]$ ergaben jedoch, wie auch bei den übrigen Techniken der peripheren Nervenrekonstruktion, deutlich heterogene Ergebnisse [2]. Die größte Erfahrung besteht heutzutage im Bereich der Reanimation des gelähmten Gesichts, wo die Technik in Kombination mit anderen Verfahren regelhaft Anwendung findet.

\section{Aktuelle Anwendungsbereiche}

Bei der End-zu-Seit-Koaption handelt es sich um eine Rekonstruktionsmöglichkeit, v. a. bei fehlendem proximalem Stumpf, die zu keiner Schädigung im Bereich des Donors führt. Als Rückzugsoption bzw. Alternative zu Nerveninterponaten sollte sie bekannt sein und im mikrochirurgischen Portfolio Berücksichtigung finden.

Einen weiteren wichtigen Versorgungsbereich stellen die Transplantation sensibler Lappenplastiken sowie die motorische Rekonstruktion durch den funktionellen Muskeltransfer dar. Durch den End-zu-Seit-Anschluss motorischer Nerven ist diese Form der Rekonstruktion möglich, ohne dass ein Verlust im Donorbereich hingenommen werden muss.

Ähnlich der anderen Techniken zur peripheren Nervenrekonstruktion wird heute allgemein die sensorische Reanimation gegenüber der motorischen als besser beurteilt. In aktuellen Studien kommen v. a. Wachstumsfaktoren, wie der IGF („,insulin-like growth factor“) zum Einsatz, um die Regenerationskraft des Nervs zu verstärken. 


\section{Korrespondenzadresse}

Prof. Dr. M. Lehnhardt

Klinik für Plastische Chirurgie und

Schwerbrandverletzte, Handchirurgiezentrum, Referenzzentrum für Gliedmaßentumoren, BG-Universitätsklinikum Bergmannsheil, Ruhr-Universität Bochum, Bürkle-de-la-Camp-Platz 1, 44789 Bochum Marcus.Lehnhardt@rub.de

\section{Einhaltung ethischer Richtlinien}

Interessenkonflikt. J. Kolbenschlag und M. Lehnhardt geben an, dass kein Interessenkonflikt besteht.

Dieser Beitrag beinhaltet keine Studien an Menschen oder Tieren.

The supplement containing this article is not sponsored by industry.

\section{Literatur}

1. Ballance CA, Ballance HA, Stewart P (1903) Remarks on the operative treatment of chronic facial palsy of peripheral origin. BMJ 1:1009-1013

2. Kettle SJA, Starritt NE, Glasby MA, Hems TEJ (2012) End-to-side nerve repair in a large animal model: how does it compare with conventional methods of nerve repair. J Hand Surg Eur 38(2):192-202

3. Landwehrs GM, Brüser P (2008) [Clinical results of terminolateral neurorrhaphy in digital nerves]. Handchir Mirkochir Plast Chir 40(5):318-321

4. Letievant $E$ (1873) Traite des sections nerveuses. Bailliere, Paris

5. Ray WZ, Mackinnon SE (2010) Management of nerve gaps: autografts, allografts, nerve transfers, and end to side neurorrhaphy. Exp Neurol 223(1):77-85

6. Viterbo F, Trindade JC, Hoshino K, Mazzoni Neto A (1992) Latero-terminal neurorrhaphy without removal of the epineural sheath. Experimental study in rats. Rev Paul Med 110(6):267-275

7. Viterbo F, Amr AH, Stipp EJ, Reis FJ (2009) End-toside-neurorraphy: past, present, future. Plast Reconstr Surg 124:351e

Trauma Berufskrankh 2014 • 16[Suppl 2]:167-168 DOI 10.1007/s10039-013-1997-7

(c) Springer-Verlag Berlin Heidelberg 2013

\section{J. Kolbenschlag $\cdot$ M. Lehnhardt \\ Funktionelle Rekonstruktion peripherer Nerven. Bedeutung der End-zu-Seit-Neuroraphie}

\section{Zusammenfassung}

End-zu-Seit-Neuroraphie. Bei fehlendem proximalem Nervenstumpf wird der distale Nervenanteil an einen gesunden Spendernerven koaptiert, um eine sowohl sensorische als auch motorische Reinervation zu erhalten. Diese Herangehensweise wurde bereits 1873 erstmals beschrieben, erlebte jedoch erst Anfang der 1990er Jahre eine breitere Anwendung. Durch Verfeinerung der Technik (epineurale Fensterung am Donornerv) konnten die Ergebnisse sowohl der sensorischen als auch der motorischen Reinervation verbessert werden.

Schlussfolgerung. Insgesamt sind die Resultate in der aktuellen Literatur heterogen, die End-zu-Seit-Neuroraphie stellt jedoch eine valide Rückzugsoption zu den in der peripheren Nervenrekonstruktion etablierten Verfahren wie der End-zu-End-Koaption mit Spendernerven oder der Nerveninterposition durch Kabelinterponate dar. Sie sollte daher im mikrochirurgischen Portfolio Berücksichtigung finden, insbesondere auch im Hinblick auf die Möglichkeiten der motorischen Rekonstruktion mittels freien Muskeltransfers.

\section{Schlüsselwörter}

Verletzungen peripherer Nerven - Operative Therapie · Neuroraphie · Axonales Sprouting . Nervale Regeneration

\section{Functional reconstruction of peripheral nerves. Importance of end-to-side neurorraphy}

\section{Abstract}

End-to-side neurorrhaphy. In end-to-side neurorrhaphy, a distal nerve segment is attached to a healthy donor nerve in an end-toside fashion. This technique is especially useful in cases were the proximal nerve stump is unavailable and allows for sensoric and motoric recovery. This technique was first described in 1873 but had not found wide application until the early 1990s. Through technical improvements (creation of an epineural window on the donor nerve), the results of sensoric as well as motoric reinervation could be improved.

Conclusion. The results presented in the literature today are heterogeneous; howev- er, end-to-side neurorrhaphy is a valid salvage procedure in case the more established methods of nerve repair (end-to-end neurorrhaphy, interposition of harvested nerves) are not feasible. Therefore, this technique should have its place in the armamentarium of $\mathrm{mi}-$ crosurgical procedures, especially considering the possibilities of reconstructing motor function through free muscle transfer.

\section{Keywords}

Peripheral nerve injuries - Operative procedures · Neurorrhaphy · Axonal sprouting $\cdot$ Nerve regeneration 